\title{
Systematic analysis reveals cis and trans determinants affecting C-to-U RNA editing in Arabidopsis thaliana
}

Duan Chu and Lai Wei ${ }^{*}$

\begin{abstract}
Background: C-to-U RNA editing is prevalent in the mitochondrial and chloroplast genes in plants. The biological functions of a fraction of C-to- $U$ editing sites are continuously discovered by case studies. However, at genome-wide level, the cis and trans determinants affecting the occurrence or editing levels of these C-to-U events are relatively less studied. What is known is that the PPR (pentatricopeptide repeat) proteins are the main trans-regulatory elements responsible for the C-to-U conversion, but other determinants especially the cis-regulatory elements remain largely uninvestigated.

Results: By analyzing the transcriptome and translatome data in Arabidopsis thaliana roots and shoots, combined with RNA-seq data from hybrids of Arabidopsis thaliana and Arabidopsis lyrata, we perform genome-wide investigation on the cis elements and trans-regulatory elements that potentially affect C-to- $U$ editing events. An upstream guanosine or double-stranded RNA (dsRNA) regions are unfavorable for editing events. Meanwhile, many genes including the transcription factors may indirectly play regulatory roles in trans.

Conclusions: The 5-prime thymidine facilitates editing and dsRNA structures prevent editing in cis. Many transcription factors affect editing in trans. Although the detailed molecular mechanisms underlying the cis and trans regulation remain to be experimentally verified, our findings provide novel aspects in studying the botanical C-to-U RNA editing events.
\end{abstract}

Keywords: C-to-U RNA editing, Cis, Trans, Regulatory, Arabidopsis, Synonyms"Missense" and "nonsynonymous".

\section{Key message}

In Arabidopsis thaliana, the 5-prime nucleotide and the RNA secondary structures affect C-to-U RNA editing in cis while many transcription factors play regulatory roles in trans.

\section{Background}

In the plant kingdom, C-to-U RNA editing is one of the most prevalent RNA modifications and is enriched in the chloroplast and mitochondrial genes [1-6]. The biological functions of particular $\mathrm{C}$-to-U editing events are discovered [7-10]. For example, phenotypic studies have associated

\footnotetext{
*Correspondence: wl@bnu.edu.cn

College of Life Sciences, Beijing Normal University, No. 19 Xinjiekouwai Street, Haidian District, Beijing, China
}

mitochondrial C-to-U editing with seed development in maize (Zea mays) and rice (Oryza sativa) [11-13]. Mechanistic study found that a particular C-to-U editing site in Oenothera gene nad1 is required for proper splicing of premRNA [14]. Early studies in sugar beet (Beta vulgaris) and tobacco (Nicotiana tabacum) found that mitochondrial Cto-U editing events were able to create translation start codons and produce functionally important proteins [15-17].

Apart from these case studies on editing function, the large-scale identification of $\mathrm{C}$-to- $\mathrm{U}$ editing sites appeared in recent years with the development of next generation sequencing (NGS) technique. Bioinformatic tools are published to systematically identify bona fide $\mathrm{C}$-to- $\mathrm{U}$ editing sites [18-21] and databases are built to collect the editing sites reported by different researchers [20,22]. 
Despite these fruitful findings in functional RNA editing and the convenience for accessing the editing sites, it remains uninvestigated that, at genome-wide level, what are the cis and trans determinants affecting the occurrence or editing levels of these C-to- $\mathrm{U}$ events? The established knowledge is that multiple factors are responsible for C-to-U RNA editing in plants, the beststudied of which is the PPR (pentatricopeptide repeat) protein [23]. However, other determinants especially the cis-regulatory elements remain largely undiscovered.

This issue could be partially resolved with the aid of next generation sequencing technique. RNA-seq (or mRNA-seq) typically sequences the fragmented reads from cellular mRNAs and could provide information on the abundance of each transcript. Ribosome profiling followed by deep sequencing [24] captures the ribosome protected mRNA fragments (about $30 \mathrm{nt}$ ), allowing a more accurate estimation of gene expression at the translatome level [25]. Thus, the cis features affecting editing sites might be parsed from the mRNA-seq data while the trans acting genes could be inferred from the genome-wide expression profile calculated from ribosome profiling data.

In this study, by analyzing the transcriptome (RNA-seq) and translatome (ribosome profiling) data in Arabidopsis thaliana roots and shoots [26, 27], combined with RNA-seq data from hybrids of Arabidopsis thaliana and Arabidopsis lyrata [28], we perform genome-wide investigation on the cis elements and trans-regulatory elements that potentially affect $\mathrm{C}$-to- $\mathrm{U}$ editing events. We find that the 5-prime nucleotide and the RNA secondary structures affect C-to-U RNA editing in cis, and transcription factors might affect editing in trans. Our findings provide novel aspects in studying the botanical C-to-U RNA editing events and should be appealing to the broad phytologists as well as RNA biologists.

\section{Results}

Identification of bona fide C-to-U RNA editing sites

Following the results of our recent study [29], using the mRNA-seq from roots and shoots of Arabidopsis thaliana, we identify $130 \mathrm{C}$-to-U RNA editing sites genome-wide, with 12 sites in chloroplast genome, 111 sites in mitochondrial genome, and 7 sites in nucleus genome ( 3 sites in chromosome 2, 1 site in chromosome 3, and 3 sites in chromosome 4). To demonstrate the reliability of the editing sites we find, we show that the $\mathrm{C}$-to- $\mathrm{U}$ alterations compose more than $80 \%$ of the total variations detected in mRNA-seq (Fig. 1a) and most of which take place in mitochondrial or chloroplast genes. One hundred thirteen of the 123 bona fide editing sites are detectable in all six mRNA-seq samples (three root samples and three shoot samples). The C-to-U editing sites have remarkably different context compared to the unedited cytidines in the genome (Fig. 1b). Most of the 5-prime nucleotides of edited cytidines are thymidine or cytidine and only a small fraction of upstream nucleotide is guanosine or adenosine, while the context of unedited cytidines is similar to the background nucleotide component (Fig. 1b).

\section{The 5-prime nucleotide of edited cytidines has great impact on editing levels}

The appearance of $\mathrm{C}$-to- $\mathrm{U}$ editing sites is context dependent as we have shown (Fig. 1b). However, it is unclear whether the nucleotide context could influence the editing level. We investigate the relationship between editing level and the nucleotide near the editing site (Fig. 1c). Among the eight positions from position -4 to position +4 (except position 0 itself), only the nucleotide at position -1 (the 5-prime nucleotide) has impact on the editing levels of focal cytidines (Fig. 1c). Editing sites with an upstream thymidine have the highest levels and the sites with an upstream guanosine have the lowest levels (Fig. 1c). This pattern might not be surprising because if a 5-prime guanosine is unfavorable for the focal cytidine to be edited, then the editing level (Fig. 1c) should be lower for the cytidines with a 5-prime guanosine.

Transcriptome-wide analysis combined with hybrids of $A$. thaliana $\times A$. lyrata reveal the effect of RNA structure on editing events

Apart from the nucleotide context, other cis elements like RNA secondary structures might potentially affect C-to-U editing events. To test this, we employ RNALfold software to determine all the structured RNA regions within each CDS (Materials and methods). Expectedly, edited positions have significantly lower fractions in structured regions compared to the unedited positions (Fig. 2a). Together with the knowledge that the editing factors PPR (pentatricopeptide repeat) proteins are likely to bind single-stranded RNAs [23], it is reasonable that the structured RNA regions are less likely to be edited.

We seek for data to verify our hypothesis. Hybrid individuals are perfect systems to study the effect of cis elements on C-to- $\mathrm{U}$ editing events because the two different parental alleles are subjected to identical trans environment. We find mRNA-seq data conducted in the hybrids of $A$. thaliana and A. lyrata [30]. With this set of data, we would only focus on the $\mathrm{C}$-to- $\mathrm{U}$ editing sites detected in chloroplast and mitochondrial genes. We combine the chloroplast and mitochondrial genomes of these two species and map the hybrid mRNA-seq data to the combined genome (Materials and methods). We extract uniquely mapped reads so that only the regions that are nonidentical between $A$. thaliana and $A$. lyrata could be covered (otherwise the read would be mapped at least twice).

Among the $123 \mathrm{C}$-to-U editing sites we previously identified, we verify two species-specific editing sites with adequate sequencing depth (Materials and methods). Two cytidines, position 362 and position 376 on gene ATMG00580 (NADH dehydrogenase subunit 4), are fully 


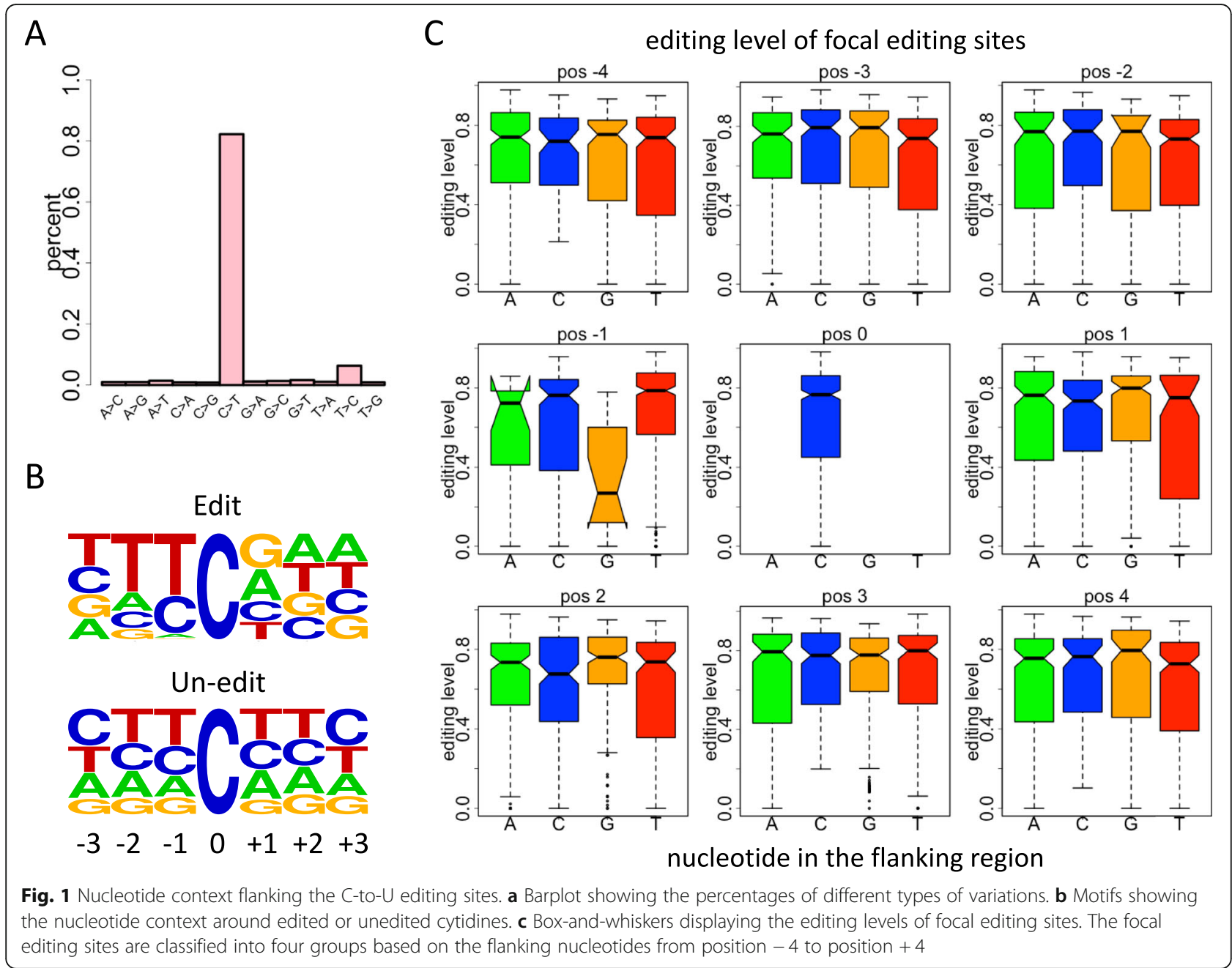

edited in A. thaliana (103 reads covered) but completely unedited in A. lyrata (31 reads covered). Interestingly, the two cytidines are located in single-stranded RNA regions in A. thaliana but in double-stranded regions in A. lyrata (Fig. 2b). This finding supports our assumption that C-to$\mathrm{U}$ editing events are favored in single-stranded RNA structures. Moreover, C-to-U event at site ATMG00580: 362 causes a missense change from ACA (Thr) to AUA (Ile) and C-to-U at site ATMG00580:376 causes a missense change from CGU (Arg) to UGU (Cys) (Fig. 2b). Thus, the different editing status between $A$. thaliana and A. lyrata might lead to the different protein products or functions of gene ATMG00580. Furthermore, it is possible that the two sites reflect the effect of mating i.e. the imprinting effect. However, this is out of the topic of this article and could be investigated in the future.

\section{Levels of different editing sites are correlated with expressions of different gene sets}

We have shown that the cis-elements like the flanking nucleotides and the RNA secondary structures could play a role in determine the occurrence or levels of Cto- $U$ editing sites. Next, we wonder whether we could find any trans regulatory factors affecting the editing levels. Although it is known that PPR proteins are responsible for C-to- $\mathrm{U}$ editing events, it does not exclude the possibility that other regulatory factors may also affect the editing levels. A previous study on mammalian A-to-I RNA editing [31] used GTEx (Genotype-Tissue Expression) mRNA-seq data and performed correlation tests between gene expressions and global editing levels, and successfully found (1) a new trans-regulatory elements AIMP2 that might affect the editing process and that (2) ADAR1 and ADAR2 positively contribute to global editing while ADAR3 plays an inhibitory role [31].

Enlightened by this study, it is conceivable that if we intend to estimate the protein level of each gene with NGS data, Ribo-seq (ribosome profiling followed by sequencing) should have stronger power than mRNA-seq. With the six mRNA-seq samples and the matched Riboseq data, we perform pairwise correlation test between the editing levels of each site in six samples and the 


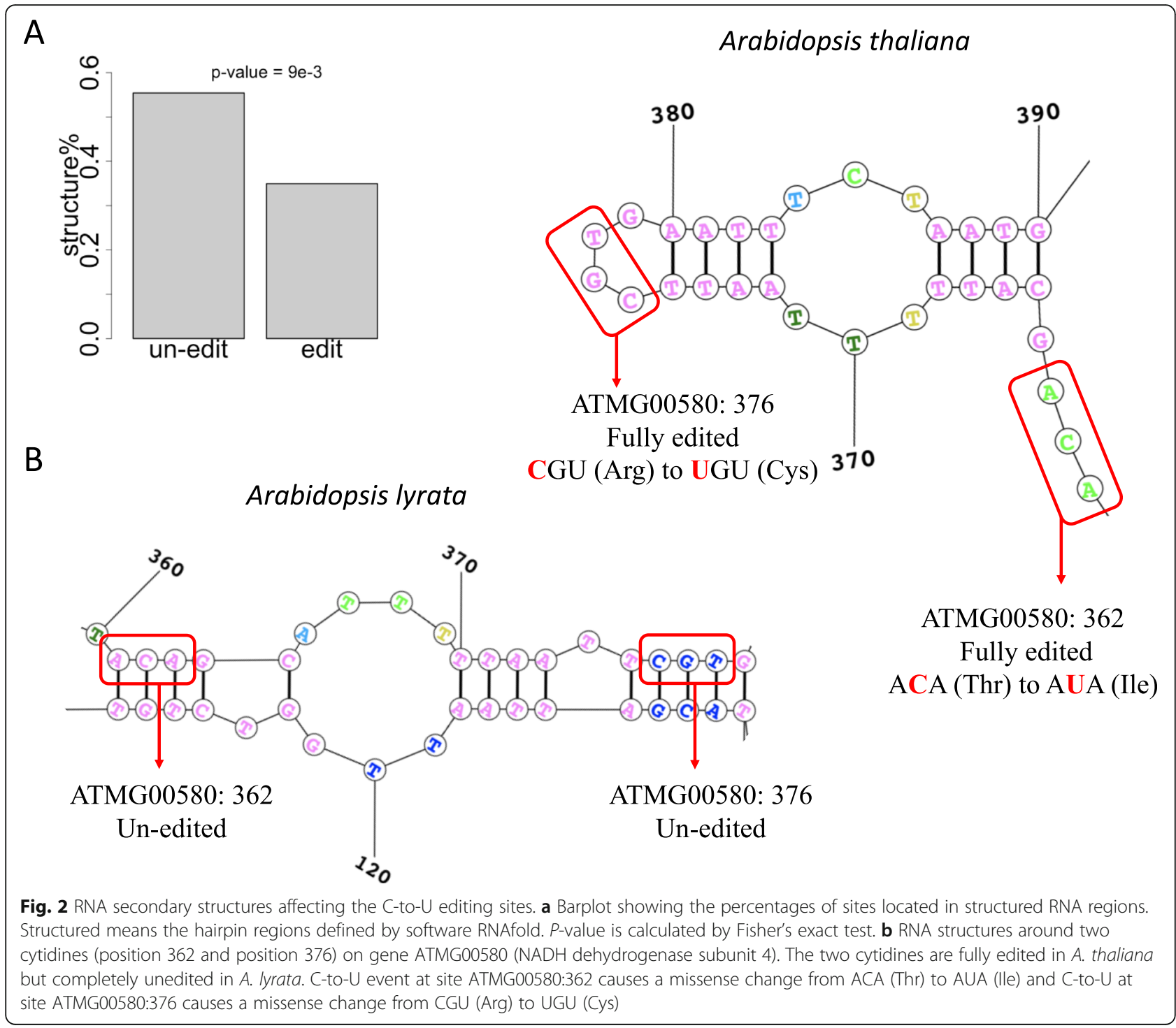

expression level (Ribo-seq) of each gene in six samples (Fig. 3a). We use the 113 out of 123 editing sites that are detectable in all six samples to correlate with the $\sim 27,000$ coding genes annotated in A. thaliana. Some sites have positive correlations with many genes while some other sites tend to show negative correlations or no correlation with gene expression (Fig. 3a). With multiple testing corrected [32] FDR $<0.05$ in the Spearman correlation, we count the numbers of genes that are significantly correlated with editing levels of each site. Because the six samples contain three root samples and three shoot samples, it is reasonable to require the expression variation within three roots/three shoots to be smaller than the variation among six samples. Let SE = "standard error of mean", based on the normal distribution of gene expression profile, we require $\mathrm{SE}_{\text {root }}<\mathrm{SE}_{\text {all }} \& \mathrm{SE}_{\text {shoot }}<\mathrm{SE}_{\text {all }}$ (root $=$ three root samples; $\quad$ shoot $=$ three shoot samples; all $=$ six samples). Under these criteria, each editing site has less than 100 correlated genes (Fig. 3b). The 113 editing sites could be generally divided into three classes. Class I sites have more positively correlated genes, class III sites have more negatively correlated genes, and class II sites have very few genes correlated (Fig. 3b). Intuitively, it seems that class I and class III sites are more highly regulated than class II sites, so we guess that the class I and class III sites might be more essential or functional. Interestingly, class I and class III sites have significantly higher editing levels (Fig. 3c) and also less variable levels (Fig. 3d) than class II sites. Moreover, class I and class III sites have higher fractions of nonsynonymous (missense) editing sites than class II (Fig. 3e). Indeed, types I, II, and III sites are of different functions. Since the number of unique genes bearing editing events is too small to perform a gene ontology analysis, we would like to list the editing genes 


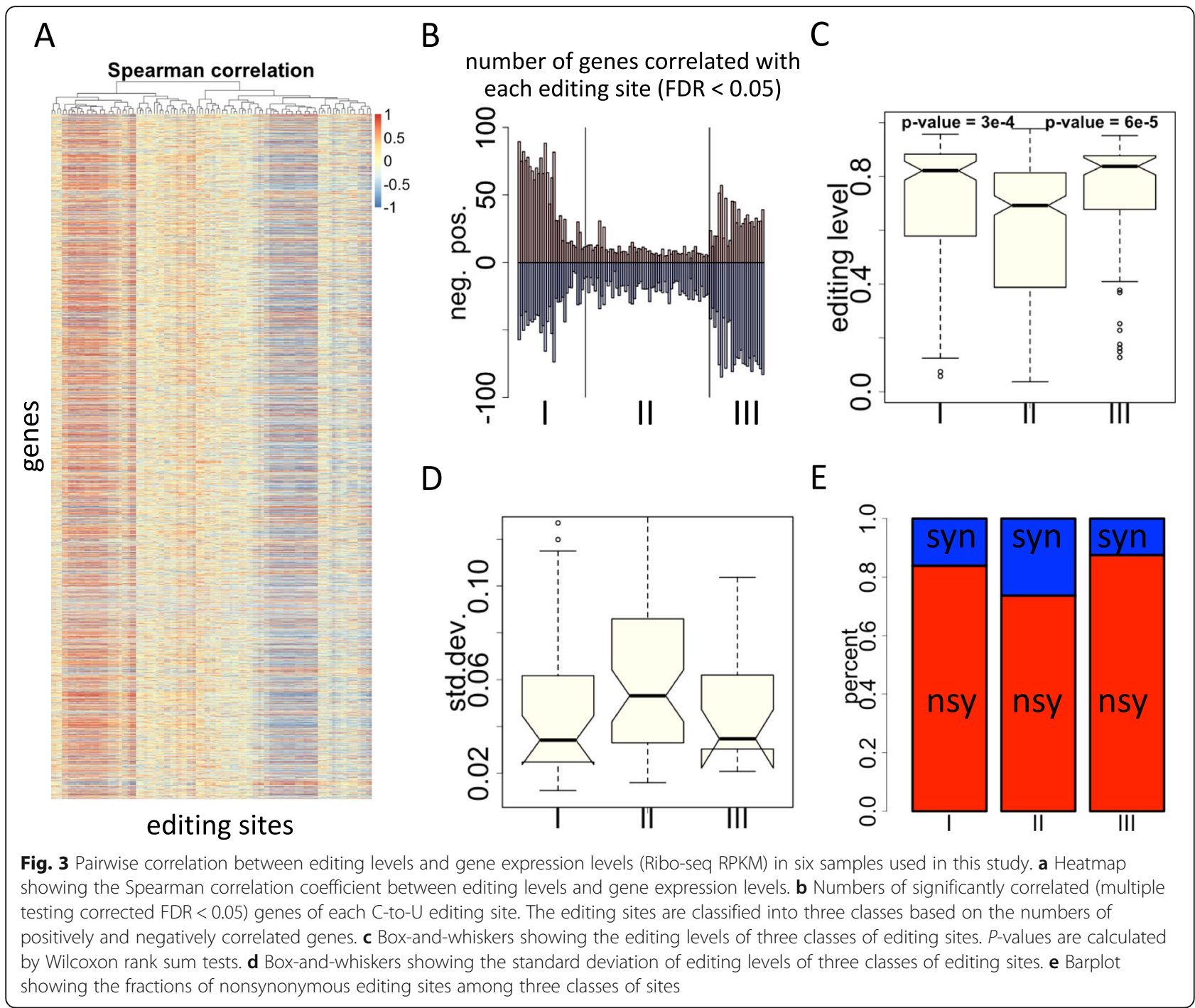

Table 1 Genes specific to type I, II, and III editing sites

\begin{tabular}{lll}
\hline Type I/IIIII site specific genes & Gene ID & Gene name \\
\hline Type I site & ATCG00740 & RPOA (RNA polymerase alpha subunit) \\
Type I site & ATCG00670 & CLPP1 (Caseinolytic protease P1) \\
Type I site & ATCG01050 & NDHD (NAD(P) H dehydrogenase complex) \\
Type I site & ATCG00040 & MATK (Maturase K) \\
Type II site & ATMG00180 & CCB452 (Cytochrome C biogenesis orf452) \\
Type II site & ATMG00690 & ORF240A (FO-ATPase subunit) \\
Type II site & ATMG00080 & RPL16 (Mitochondrial ribosomal protein L16) \\
Type II site & ATMG00640 & ORF25 (Plant b subunit of mitochondrial ATP synthase) \\
Type II site & ATCG00180 & RPOC1 (RNA polymerase beta' subunit-1) \\
Type II site & ATCG00300 & PSBZ (Photosystem II subunit) \\
Type III site & ATMG01170 & ATP6-2 (ATPase subunit 6) \\
\hline
\end{tabular}


that are specific to type I, II, III sites. In other words, if a gene has both type I and type II sites, then this gene is excluded. In Table 1, we see that the genes specific to type I sites are chloroplast genes (gene ID beginning with ATCG, $\mathrm{C}$ stands for chloroplast) while the genes specific to type II and type III sites are mainly mitochondrial genes (gene ID beginning with ATMG, M stands for mitochondrial). This indicates the differential functions of the genes as well as the editing sites located on them.

Transcription factors show the strongest correlations with editing levels in different samples

The above paragraph focuses on the classification of editing sites according to their correlations with Ribo-seq gene expression levels. Here we wonder what kind of genes have the strongest correlation with levels of editing sites. We first define two sets of genes, $\mathrm{n} 1=$ genes with significant positive correlations with at least 10 editing sites, $\mathrm{n} 2=$ genes with significant negative correlations with at least 10 editing sites. Next, we define three classes of genes according to $\mathrm{n} 1$ and $\mathrm{n} 2$. The definition is as follows. "Positive" genes = set difference $(\mathrm{n} 1-\mathrm{n} 2)=\mathrm{n} 1 \cap \neg \mathrm{n} 2$, "negative" genes = set difference $(\mathrm{n} 2-\mathrm{n} 1)=\mathrm{n} 2 \cap \neg \mathrm{n} 1$, "both" genes = intersection $(\mathrm{n} 1 \& \mathrm{n} 2)=\mathrm{n} 1 \cap \mathrm{n} 2$. With this definition, it is understandable that "positive" genes (331 genes) represent the genes with the greatest numbers of positively correlated editing sites, "negative" genes (479 genes) represent the genes with the greatest numbers of negatively correlated editing sites, and "both" genes (433 genes) have positive or negative correlations with different sets of editing sites.

Interestingly, the functional annotation shows that all these three sets of genes are significantly enriched in transcription factors (Fig. 4a). We illustrate two examples of transcription factors from positive genes and negative genes respectively (Fig. 4b). The $\mathrm{X}$-axis represents six samples we use, and is ranked by increasing

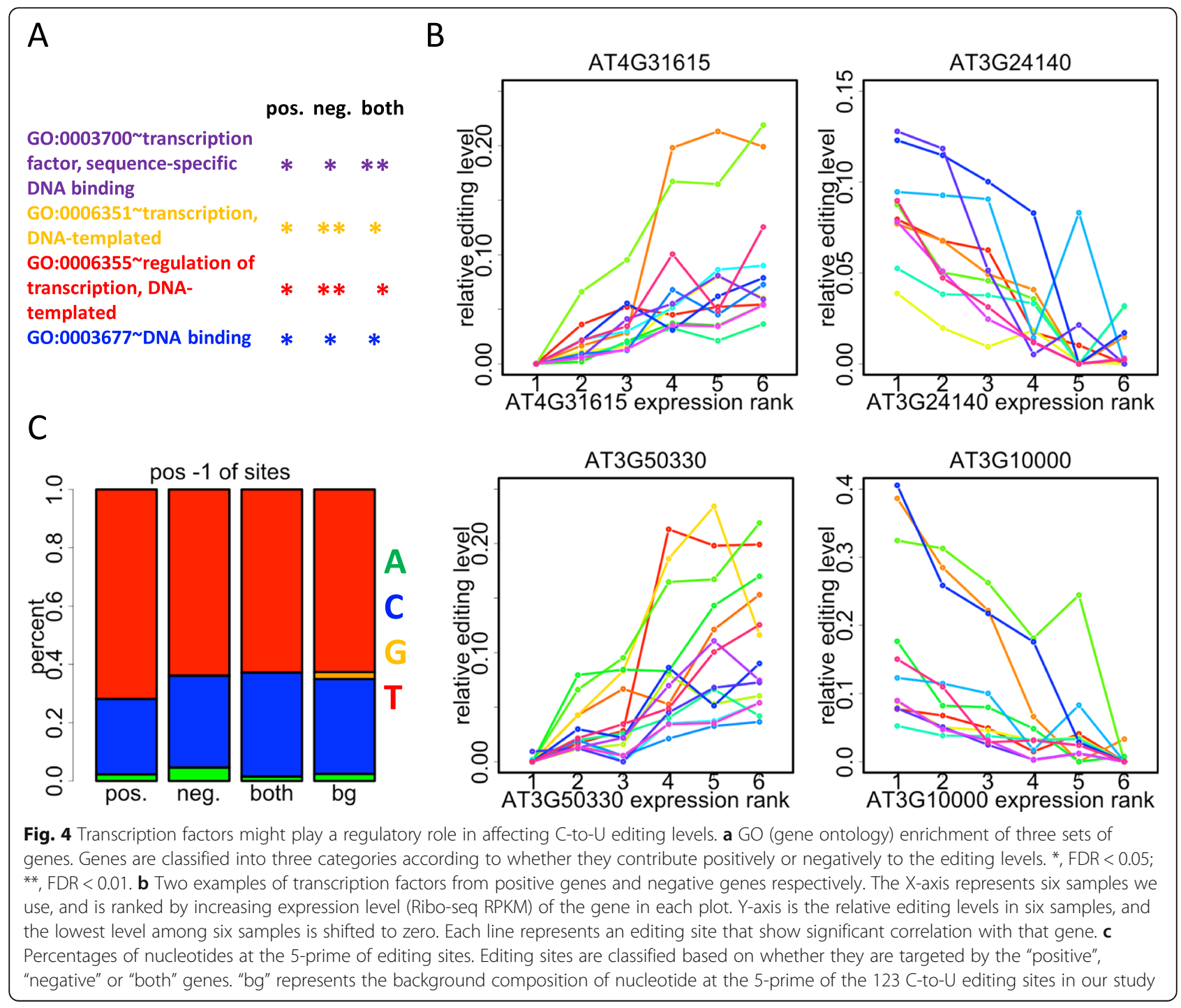


expression level (Ribo-seq RPKM) of the gene in each plot. Y-axis is the relative editing levels in six samples, and the lowest level among six samples is shifted to zero. Each colorful line represents an editing site that show significant correlation with that gene (Fig. 4b). There is a clear trend that the two positive genes AT4G31615 and AT3G50330 contribute positively to the editing level while the two negative genes AT3G24140 and AT3G10000 show the opposite trend. AT4G31615 (REM35) is a transcriptional factor B3 family protein located in chloroplast, AT3G50330 is a bHLH transcription factor located in nucleus, AT3G24140 is a basic helix-loop-helix transcription factor located in nucleus, and AT4G10000 is located in chloroplast. The possibilities of how transcription factors affect the organelle editing would be discussed later.

As previous study proposed [33] or indicated from our data, different factors (genes) might be responsible for different editing sites. Based on this assumption, we pool the significantly correlated editing sites of positive, negative and both genes, respectively, and investigate the component of their 5-prime nucleotides (Fig. 4c). There is a higher fraction of thymidine at the 5-prime of editing sites (compared to background) which are correlated with positive genes (Fig. 4c). This result associates the trans-regulatory elements with the cis elements, suggesting that the editing sites controlled by different factors might have different sequence features.

At this moment, it is difficult to figure out how transcription factors could affect C-to-U RNA editing events, and there is no direct evidence to show that the trans determinants like transcription factors are related to the sequence motif around C-to- $\mathrm{U}$ editing sites. However, we stress the idea that the editing events might be indirectly affected by various factors apart from the PPR proteins. Moreover, the trans-regulatory elements and cis elements might be interconnected and it is not necessary to tear them apart in the functional studies as if they were independent.

\section{Discussion}

Mutations act as the source for natural selection. Missense mutations lead to amino acid alterations and are intuitively subjected to selection force [34, 35]. Synonymous mutations do not change amino acids but they could be selected due to their impacts on mRNA splicing [36], codon optimality [37, 38], GC content [39], mRNA translation [40], and codon order [41]. Even the noncoding mutations could have a functional consequence by affecting microRNA targeting [42]. Since C-to-U RNA editing has similar consequences to mutations, these editing events must be carefully regulated by the organisms.

Apart from the PPR proteins as direct catalyzer of Cto-U conversion, other indirect trans or cis features may also contribute to the editing events. By analyzing the transcriptome and translatome data in Arabidopsis thaliana roots and shoots [26], combined with RNA-seq data from hybrids of Arabidopsis thaliana and Arabidopsis lyrata [28], we demonstrate that an upstream guanosine is unfavorable for the occurrence of $\mathrm{C}$-to- $\mathrm{U}$ conversion. Surprisingly, this sequence context found for C-to- $\mathrm{U}$ editing sites is extremely similar to that of A-to-I RNA editing sites in animals [43-45], where a guanosine upstream of the edited adenosine is strongly avoided. In animals, the preference of sequence context around Ato-I editing sites is caused by the biochemical property of ADAR proteins [46]. Whether the context of C-to- $\mathrm{U}$ sites in plants is related to their catalytic enzyme remains to be investigated.

We also reveal that the double-stranded RNA regions are unfavorable for editing events. The structural basis we found could be supported by the fact that editing factors PPR proteins are likely to bind single-stranded RNAs [23]. More intriguingly, the animal A-to-I RNA editing events are also strongly affected by RNA secondary structures [47, 48] and the imperfectly-paired double-stranded RNAs are best substrates of ADAR. In plants, we found that C-to-U RNA editing sites are enriched in single-stranded (unstructured) RNA regions, which is opposite to the case of A-to-I editing. The structural basis for C-to-U or A-to-I RNA editing might not be a coincidence. If the editing events take place randomly in all regions without any specificity, then they are most likely to be non-adaptive and should be purged by natural selection. Note that the definition of doublestranded RNA is ambiguous for some loop regions in structured RNAs. It is debatable whether the loops belong to single- or double-stranded RNAs. Therefore, it is possible that the bonds in double-stranded RNAs prevent editing.

Meanwhile, by performing genome-wide correlation tests, we also find that different transcription factors might contribute positively or negatively to different editing sites. As we have mentioned, the previous study on mammalian A-to-I RNA editing [31] using GTEx mRNA-seq data has successfully found a new transregulatory elements AIMP2 that might affect the editing process. The cited literature also used correlation tests. Moreover, compared to mRNA-seq data, the Ribo-seq we use in our study might have stronger power to resemble the final protein amount. Indeed, transcription factors interact with DNA while the C-to- $\mathrm{U}$ editing events take place at RNA level. To discuss why nucleus genes could regulate the cytoplasmic editing, we raise some possibilities without further validation. First, the regulation is indirect. The transcription factors affect the gene expression of other related genes and those genes regulate RNA editing. Second, if the transcription factors indirectly regulate the expression level of editing genes, 
then there might be feedback loops controlling the editing status of those editing genes. Although the editing process does not take place in nucleus, the transcription factors might change editing levels via controlling the amount of editing transcripts. At this moment, it is hard to attribute the editing level fluctuations to the transcription factors. The molecular mechanisms remain to be investigated.

Last but not least, it is hypothesized that C-to-U RNA editing in plants could be designed for reversing the potentially unfavorable T-to-C DNA mutations [49] and therefore the editing levels should be constantly high to mimic the DNA mutation. Our results show that although the editing levels are fluctuating across samples, the range of the most variable levels is usually less than $20 \%$ as shown in Fig. 4b. Thus, our study does not conflict with established knowledges. We add new aspects in depicting the C-to-U RNA editing mechanisms in plants and would be appealing to the broad plant biologists.

\section{Conclusions}

Our study reveals that the 5-prime nucleotide and the RNA secondary structures affect C-to-U RNA editing in cis. An upstream guanosine or double-stranded RNA regions are unfavorable for editing events. Meanwhile, many genes including the transcription factors play regulatory roles in trans. Different transcription factors might contribute positively or negatively to different editing sites.

\section{Methods}

\section{Data collection}

The reference genome and CDS sequences of Arabidopsis thaliana were downloaded from TAIR database. The TAIR 9 version of annotation was used. The mRNA-seq and Ribo-seq data of Arabidopsis thaliana (three replicates for roots and shoots) were retrieved from a previous study [26]. As described by the paper, the Arabidopsis Columbia-0 (abbr. Col-0) seeds (around 1500 seeds per vessel) were surface sterilized and imbibed at $4{ }^{\circ} \mathrm{C}$ for 2 days. They were grown hydroponically on fine nylon mesh supported by a customized rack in Magenta vessel GA-7-3 (Sigma; V8380) with filtered sterile liquid media and shaken at $85 \mathrm{rpm}$ under $16-\mathrm{h}$ light $\left(110-115 \mu \mathrm{mol} \mathrm{m}{ }^{-2} \cdot \mathrm{s}^{-1}\right.$ from cool white fluorescent bulbs) and 8 -h dark at $22^{\circ} \mathrm{C}$ [26]. The tissues were collected from 4-day old seedlings.

The RNA-seq data from hybrids of Arabidopsis thaliana and Arabidopsis lyrata were generated by a previous study [28] under accession number SRP073606 (100 bp, pair-ended). In the cross experiment, $A$. thaliana was used as a mother (Col-0, obtained from the Arabidopsis Biological Resource Center, ABRC, USA) and A. lyrata was used as a father (Arabidopsis lyrata ssp. lyrata genotype MN47). Hybrid seeds were germinated and grown on germination medium containing Murashige and Skoog salts, $1 \%$ sucrose, and $0.8 \%$ agar. The plants were stratified for 5 days at $4{ }^{\circ} \mathrm{C}$, and then grown for 4 weeks in a growth chamber at $22^{\circ} \mathrm{C} / 16^{\circ} \mathrm{C}$ under $16 \mathrm{~h} \mathrm{light} / 8 \mathrm{~h}$ dark. Then, different treatments were done to the hybrid samples. For dehydration, plants were removed from the agar and dehydrated in plastic dishes for $1 \mathrm{~h}$ at $22^{\circ} \mathrm{C}$ under dim light $\left(0.7 \pm 0.8 \mathrm{mmol} \mathrm{s}^{-1} \mathrm{~m}^{-1}\right)$. For cold exposure, plants were grown under dim light $(0.7 \pm 0.8$ mmol s${ }^{-1} \mathrm{~m}^{-1}$ ) at $4{ }^{\circ} \mathrm{C}$ for $1 \mathrm{~h}$. Leaf samples of plants growing in non-stressful conditions (standard treatment) were collected on 4 week-old plants grown at $22^{\circ} \mathrm{C}$. To control for circadian changes in gene expression, all samples were collected at 12:00 pm. In the mRNA-seq data analysis, to increase the power in detecting RNA editing sites, all the mRNA-seq libraries from different conditions including the control samples are pooled.

\section{Mapping the NGS reads}

For the A. thaliana data, we map the mRNA-seq reads to the reference CDS sequences using Bowtie2 [30]. For the hybrids of $A$. thaliana and A. lyrata, we only focus on the $\mathrm{C}$-to- $\mathrm{U}$ editing sites detected in chloroplast and mitochondrial genes.

The $A$. lyrata genome (CDS sequences) is downloaded from the Ensembl website with the following code (wget $\mathrm{ftp}: / / \mathrm{ftp}$.ensemblgenomes.org/pub/plants/release-44/ fasta/arabidopsis_lyrata/cds/Arabidopsis_lyrata.v.1.0.cds. all.fa.gz). We combine the chloroplast and mitochondrial genomes of these two species and map the hybrid mRNA-seq data to the combined genome with Bowtie2. We extract uniquely mapped reads so that only the regions that are non-identical between $A$. thaliana and $A$. lyrata could be covered (otherwise the read would be mapped at least twice). The uniquely mapped reads are extracted using SAMtools [50].

\section{Variant calling}

The variant calling process is accomplished by SAMtools "mpileup". The minimum mapping quality of reads is set to be 20 (parameter $-\mathrm{q} 20$ ) and minimum base quality is set to be 30 (parameter $-\mathrm{Q} 30$ ) to increase the accuracy of variation sites. The output file of mpileup is "vcf" format, which contains one variation site per line. The information for each variation site includes total depth on each site, the reference allele count and each alternative allele count. Sites with more than one variation type are discarded. Variation level $=$ alternative allele count $/$ total depth. Take C-to-U RNA editing sites for instance, editing level $=\mathrm{T} /(\mathrm{C}+\mathrm{T})$. To avoid the false positive variants caused by technical limitations, the variant sites with levels higher than 0.05 and with at least 10 covered reads are maintained [51-53]. Note that one gene could have multiple isoforms so that some variation sites appearing 
in different isoform might belong to the same genomic location. Thus, only the longest CDS was used for variant calling to avoid the redundancy.

In the RNA-seq of hybrid, with the uniquely mapped reads, we have verified two species-specific sites with adequate sequencing depth. Two cytidines, position 362 and position 376 on gene ATMG00580 (NADH dehydrogenase subunit 4), are fully edited in A. thaliana (103 reads covered) and completely unedited in A. lyrata (31 reads covered).

\section{RNA structure}

We use RNALfold [54] to determine the regions with RNA secondary structures. Each CDS sequence is input to the software. With default parameters, the output file would contain the positions of all the secondary structures within the input sequence. We merge the structured regions within each CDS. The length of structured regions divided by the length of each CDS is the structure\% for each gene. Our loose criterion considers all the structured regions with Z-score $<0$ reported by the software. All the CDSs we used contain 33.2 million bases and all the structured regions under our criterion contain 18.4 million bases $(18.4 / 33.2=55 \%)$. Among the $123 \mathrm{C}$-to-U editing sites we identified, 43 of them are located in structured regions $(43 / 123=35 \%)$.

The diagram of RNA structure is accomplished with online tools RNA structure (http://rna.urmc.rochester. edu/RNAstructureWeb/Servers/Predict1/Predict1.html). The only input message needed is the nucleotide sequence.

\section{Gene expression analysis}

The expression level of a gene is defined as RPKM (reads per kilobase per million mapped reads). In the correlation test between editing levels and gene expression, the RPKM of Ribo-seq reads are used to represent the expression of a gene.

\section{Functional annotation}

The functional annotation of the genes IDs is performed using the online software DAVID [55].

\section{Statistical analysis and code availability}

All statistical analyses (for example, correlation tests) and the graphic work were conducted in $\mathrm{R}$ environment (http://www.R-project.org/). All codes used in the analyses are available under request.

\section{Abbreviations}

mRNA: Messenger RNA; CDS: Coding sequence; NGS: Next generation sequencing; nsy: Nonsynonymous; syn: Synonymous

\section{Acknowledgements}

At this COVID-19 time, we thank all medical workers fighting in the frontline. Without their sacrifice, our safety could not be guaranteed. We also thank the lab members who have given suggestions to this article.

\section{Authors' contributions}

LW designed and supervised this research. Both DC and LW analyzed the data. DC and LW wrote this article. The author(s) read and approved the final manuscript.

\section{Funding}

This research was financially supported by the National Natural Science Foundation of China (Grant no. 31770213). The funders had no role in study design, data collection and analysis, decision to publish, or preparation of the manuscript.

\section{Availability of data and materials}

All data used in this study are public data, the sources or links of which are provided in the Materials and Methods.

Ethics approval and consent to participate

Not applicable.

\section{Consent for publication}

Not applicable.

\section{Competing interests}

The authors declare they have no competing interests.

Received: 13 June 2020 Accepted: 26 August 2020

Published online: 03 September 2020

\section{References}

1. Mulligan RM, Williams MA, Shanahan MT. RNA editing site recognition in higher plant mitochondria. J Hered. 1999;90(3):338-44.

2. Rajasekhar VK, Mulligan RM. Rna editing in plant-mitochondria - alphaphosphate is retained during $\mathrm{C}$-to- $\mathrm{U}$ conversion in messenger-Rnas. Plant Cell. 1993;5(12):1843-52.

3. Gray MW. RNA editing in plant mitochondria: 20 years later. IUBMB Life. 2009;61(12):1101-4.

4. Gray MW, Covello PS. Rna editing in plant-mitochondria and chloroplasts. FASEB J. 1993;7(1):64-71.

5. Tang W, Luo C. Molecular and functional diversity of RNA editing in plant mitochondria. Mol Biotechnol. 2018;60(12):935-45.

6. Yu W, Schuster W. Evidence for a site-specific Cytidine deamination reaction involved in C to U Rna editing of plant-mitochondria. J Biol Chem. 1995; 270(31):18227-33.

7. Takenaka M. MEF9, an E-subclass Pentatricopeptide repeat protein, is required for an RNA editing event in the nad7 transcript in mitochondria of Arabidopsis. Plant Physiol. 2010;152(2):939-47.

8. Takenaka M, Neuwirt J, Brennicke A. Complex cis-elements determine an RNA editing site in pea mitochondria. NAR. 2004;32(14):4137-44.

9. Takenaka M, Verbitskly D, van der Merwe JA, Zehrmann A, Brennicke A. The process of RNA editing in plant mitochondria. Mitochondrion. 2008:8(1):3546.

10. Tang JW, Kobayashi K, Suzuki M, Matsumoto S, Muranaka T. The mitochondrial PPR protein LOVASTATIN INSENSITIVE 1 plays regulatory roles in cytosolic and plastidial isoprenoid biosynthesis through RNA editing. Plant J. 2010;61(3):456-66.

11. Li XJ, Zhang YF, Hou MM, Sun F, Shen Y, Xiu ZH, Wang XM, Chen ZL, Sun SSM, Small I, et al. Small kernel 1 encodes a pentatricopeptide repeat protein required for mitochondrial nad7 transcript editing and seed development in maize (Zea mays) and rice (Oryza sativa). Plant J. 2014;79(5): 797-809.

12. Liu YJ, Xiu ZH, Meeley R, Tan BC. Empty Pericarp5 encodes a Pentatricopeptide repeat protein that is required for mitochondrial RNA editing and seed development in maize. Plant Cell. 2013;25(3):868-83.

13. Sun F, Wang XM, Bonnard G, Shen Y, Xiu ZH, Li XJ, Gao DH, Zhang ZH, Tan BC. Empty pericarp7 encodes a mitochondrial E-subgroup pentatricopeptide repeat protein that is required for $\mathrm{ccmF}(\mathrm{N})$ editing, 
mitochondrial function and seed development in maize. Plant J. 2015;84(2): 283-95.

14. Borner GV, Morl M, Wissinger B, Brennicke A, Schmelzer C. Rna editing of a group-ii intron in Oenothera as a prerequisite for splicing. Mol Gen Genet. 1995;246(6):739-44.

15. Kubo T, Yamamoto MP, Mikami T. The nad4L-orf25 gene cluster is conserved and expressed in sugar beet mitochondria. Theor Appl Genet. 2000;100(2):214-20.

16. Onodera Y, Yamamoto MP, Kubo T, Mikami T. Heterogeneity of the atp6 presequences in normal and different sources of male-sterile cytoplasms of sugar beet. J Plant Physiol. 1999;155(4-5):656-60.

17. Kudla J, Igloi G, Metzlaff M, Hagemann R, Kossel H. Rna editing in tobacco chloroplasts leads to the formation of a translatable Psbl messenger-Rna by a C to U substitution within the initiation codon. EMBO J. 1992;11(3):1099103.

18. Lo Giudice C, Hernandez I, Ceci LR, Pesole G, Picardi E. RNA editing in plants: a comprehensive survey of bioinformatics tools and databases. Plant Physiol Biochem. 2019;137:53-61.

19. Du PF, Jia LY, Li YD. CURE-chloroplast: a chloroplast C-to-U RNA editing predictor for seed plants. BMC Bioinform. 2009;10.

20. Li M, Xia L, Zhang YS, Niu GY, Li MW, Wang P, Zhang Y, Sang J, Zou D, Hu $\mathrm{SN}$, et al. Plant editosome database: a curated database of RNA editosome in plants. NAR. 2019;47(D1):D170-4.

21. Picardi E, Pesole G. REDItools: high-throughput RNA editing detection made easy. Bioinformatics. 2013;29(14):1813-4.

22. Lo Giudice C, Pesole G, Picardi E. REDIdb 3.0: A Comprehensive Collection of RNA Editing Events in Plant Organellar Genomes. Front Plant Sci. 2018:9.

23. Yin P, Li QX, Yan CY, Liu Y, Liu JJ, Yu F, Wang Z, Long JF, He JH, Wang HW, et al. Structural basis for the modular recognition of single-stranded RNA by PPR proteins. Nature. 2013;504(7478):168.

24. Ingolia NT, Ghaemmaghami S, Newman JR, Weissman JS. Genome-wide analysis in vivo of translation with nucleotide resolution using ribosome profiling. Sci. 2009;324(5924):218-23.

25. Chu D, Wei L. Characterizing the heat response of Arabidopsis thaliana from the perspective of codon usage bias and translational regulation. J Plant Physiol. 2019;240:153012

26. Hsu PY, Calviello L, Wu HYL, Li FW, Rothfels CJ, Ohler U, Benfey PN. Superresolution ribosome profiling reveals unannotated translation events in Arabidopsis. Proc Natl Acad Sci U S A. 2016;113(45):E7126-35.

27. Chu $D$, Wei $L$. Reduced $C$-to-U RNA editing rates might play a regulatory role in stress response of Arabidopsis. J Plant Physiol. 2020;244.

28. He F, Arce AL, Schmitz G, Koornneef M, Novikova P, Beyer A, de Meaux J. The footprint of polygenic adaptation on stress-responsive Cis-regulatory divergence in the Arabidopsis genus. Mol Biol Evol. 2016;33(8):2088-101.

29. Chu D, Wei L. The chloroplast and mitochondrial C-to-U RNA editing in Arabidopsis thaliana shows signals of adaptation. Plant Direct. 2019;3(9): e00169.

30. Langmead B, Salzberg SL. Fast gapped-read alignment with bowtie 2. Nat Methods. 2012;9(4):357-U354.

31. Tan MH, Li Q, Shanmugam R, Piskol R, Kohler J, Young AN, Liu Kl, Zhang R, Ramaswami G, Ariyoshi K, et al. Dynamic landscape and regulation of RNA editing in mammals. Nature. 2017;550(7675):249-54.

32. Benjamini $Y$, Hochberg Y. Controlling the false discovery rate - a practical and powerful approach to multiple testing. J Royal Stat Soc Series BMethodol. 1995;57(1):289-300.

33. Karcher D, Bock R. Temperature sensitivity of RNA editing and intron splicing reactions in the plastid ndhB transcript. Curr Genet. 2002;41(1):4852.

34. Becerra-Flores M, Cardozo T. SARS-CoV-2 viral spike G614 mutation exhibits higher case fatality rate. Int J Clin Pract. 2020:e13525.

35. Chu D, Wei L. Nonsynonymous, synonymous and nonsense mutations in human cancer-related genes undergo stronger purifying selections than expectation. BMC Cancer. 2019;19(1):359.

36. Supek F, Minana B, Valcarcel J, Gabaldon T, Lehner B. Synonymous mutations frequently act as driver mutations in human cancers. Cell. 2014; 156(6):1324-35.

37. Chu D, Wei L. Genome-wide analysis on the maize genome reveals weak selection on synonymous mutations. BMC Genomics. 2020;21(1):333.

38. Grosjean H, de Crecy-Lagard V, Marck C. Deciphering synonymous codons in the three domains of life: co-evolution with specific tRNA modification enzymes. FEBS Lett. 2010;584(2):252-64.
39. Wei L. Selection on synonymous mutations revealed by 1135 genomes of Arabidopsis thaliana. Evol Bioinformatics Online. 2020;16:1176934320916794.

40. Tarrant D, von der Haar T. Synonymous codons, ribosome speed, and eukaryotic gene expression regulation. Cell Mol Life Sci. 2014;71(21):4195206.

41. Chu D, Wei L. Parsing the synonymous mutations in the maize genome: isoaccepting mutations are more advantageous in regions with codon cooccurrence bias. BMC Plant Biol. 2019;19(422). https://doi.org/10.1186/ s12870-019-2050-1.

42. Guo X, Gui Y, Wang Y, Zhu QH, Helliwell C, Fan L. Selection and mutation on microRNA target sequences during rice evolution. BMC Genomics. 2008; 9:454.

43. Buchumenski I, Bartok O, Ashwal-Fluss R, Pandey V, Porath HT, Levanon EY, Kadener S. Dynamic hyper-editing underlies temperature adaptation in drosophila. PLoS Genet. 2017;13(7):e1006931.

44. Liscovitch-Brauer N, Alon S, Porath HT, Elstein B, Unger R, Ziv T, Admon A, Levanon EY, Rosenthal JJC, Eisenberg E: Trade-off between Transcriptome plasticity and genome evolution in cephalopods. Cell 2017, 169(2):191-202 e111.

45. Porath HT, Knisbacher BA, Eisenberg E, Levanon EY. Massive A-to-I RNA editing is common across the Metazoa and correlates with dsRNA abundance. Genome Biol. 2017;18(1):185.

46. Savva YA, Rieder LE. Reenan RA. The ADAR protein family. Genome Biol. 2012;13(252). https://doi.org/10.1186/gb-2012-13-12-252.

47. Bass $B L$, Weintraub $H$. A developmentally regulated activity that unwinds Rna duplexes. Cell. 1987:48(4):607-13.

48. Higuchi M, Single FN, Kohler M, Sommer B, Sprengel R, Seeburg PH. Rna editing of Ampa receptor subunit Glur-B - a base-paired intron-exon structure determines position and efficiency. Cell. 1993;75(7):1361-70.

49. Edera AA, Gandini CL, Sanchez-Puerta MV. Towards a comprehensive picture of C-to-U RNA editing sites in angiosperm mitochondria. Plant Mol Biol. 2018;97(3):215-31.

50. Li H, Handsaker B, Wysoker A, Fennell T, Ruan J, Homer N, Marth G, Abecasis G, Durbin R, Proc GPD. The sequence alignment/map format and SAMtools. Bioinformatics. 2009;25(16):2078-9.

51. Ramaswami G, Zhang R, Piskol R, Keegan LP, Deng P, O'Connell MA, Li JB. Identifying RNA editing sites using RNA sequencing data alone. Nat Methods. 2013;10(2):128-32.

52. Yu Y, Zhou H, Kong Y, Pan B, Chen L, Wang H, Hao P, Li X. The landscape of A-to-I RNA Editome is shaped by both positive and purifying selection. PLoS Genet. 2016;12(7):e1006191.

53. Peng ZY, Cheng YB, Tan BCM, Kang L, Tian ZJ, Zhu YK, Zhang WW, Liang Y, Hu XD, Tan XM, et al. Comprehensive analysis of RNA-Seq data reveals extensive RNA editing in a human transcriptome. Nat Biotechnol. 2012;30(3): 253.

54. Hofacker IL. Vienna RNA secondary structure server. Nucleic Acids Res. 2003; 31(13):3429-31.

55. Jiao X, Sherman BT, Huang da W, Stephens R, Baseler MW, Lane HC, Lempicki RA, DAVID WS. A stateful web service to facilitate gene/protein list analysis. Bioinformatics. 2012;28(13):1805-6.

\section{Publisher's Note}

Springer Nature remains neutral with regard to jurisdictional claims in published maps and institutional affiliations.

Ready to submit your research? Choose BMC and benefit from:

- fast, convenient online submission

- thorough peer review by experienced researchers in your field

- rapid publication on acceptance

- support for research data, including large and complex data types

- gold Open Access which fosters wider collaboration and increased citations

- maximum visibility for your research: over $100 \mathrm{M}$ website views per year

At BMC, research is always in progress.

Learn more biomedcentral.com/submissions 